GA-A24185

\title{
MODELING A RESISTIVE WALL MODE CONTROL SYSTEM OF THE BANG-BANG TYPE
}

\author{
T.H. JENSEN
}

JANUARY 2003 


\section{DISCLAIMER}

This report was prepared as an account of work sponsored by an agency of the United States Government. Neither the United States Government nor any agency thereof, nor any of their employees, makes any warranty, express or implied, or assumes any legal liability or responsibility for the accuracy, completeness, or usefulness of any information, apparatus, product, or process disclosed, or represents that its use would not infringe privately owned rights. Reference herein to any specific commercial product, process, or service by trade name, trademark, manufacturer, or otherwise, does not necessarily constitute or imply its endorsement, recommendation, or favoring by the United States Government or any agency thereof. The views and opinions of authors expressed herein do not necessarily state or reflect those of the United States Government or any agency thereof. 


\title{
MODELING A RESISTIVE WALL MODE CONTROL SYSTEM OF THE BANG-BANG TYPE
}

\author{
T.H. JENSEN
}

This is a preprint of a paper to be submitted for publication in Phys. Plasmas.

\author{
Work supported by \\ the U.S. Department of Energy under \\ Grant No. DE-FG03-95ER54309
}




\section{ABSTRACT}

Feedback stabilization of the resistive wall mode is usually accomplished by using linear amplifiers. In this paper a study is made of a possibility of controlling resistive wall modes using switches instead of linear amplifiers, i.e., using bang-bang control. The motivation is that bangbang control systems may be cheaper than conventional feedback systems. A distinct disadvantage of the bang-bang system is complexity due to its inherent nonlinearity. Further studies, particularly of engineering issues, are needed to determine the attractiveness of a bangbang system. 



\section{INTRODUCTION}

Certain instabilities (resistive wall modes) of a magnetically confined plasma would be stabilized if the plasma were surrounded by a wall of infinite conductivity. When the surrounding wall has a finite conductivity, the plasma remains unstable, but the growth rates of such instabilities are reduced to the inverse of a time mainly determined by the resistance of the wall. Usually this "resistive wall time" is much larger than timescales characteristic of the plasma itself, such as Alfvén and soundwave timescales. The resistive wall time is often so long that it is practical to make the wall appear conducting to the plasma by driving currents in external coils so that magnetic fields associated with unstable modes are not allowed to penetrate the wall.

Commonly, linear amplifiers are used for driving these currents in external coils as described in Refs. 1-9. This paper describes a study of a control system which utilizes switches instead of amplifiers; such control systems are often called bang-bang control systems 10 .

The point of this paper, namely considering a bang-bang control system for stabilizing the resistive wall mode, is to show that there exist perhaps better possibilities for stabilizing resistive wall modes then the conventional ones utilizing linear amplifiers. While methods of stabilization using linear amplifiers are well explored, bang-bang control system have received little attention. This paper deals with just one example of a bang-bang control system. There must be many different ways of realizing a bang-bang control system, therefore the one described here is probably not "optimal". One way of defining an "optimal" system is as one of lowest cost among systems with equal stabilization properties.

A recent study of bang-bang control was made by Tian, Tadé, and Levy ${ }^{11}$. For the present study it was assumed, unlike for the study of Rev. 11, that some time is needed for making the decision on and implementation of which switch position should be used. 
The bang-bang system has a definite disadvantage in that it is nonlinear. It appears that little is known analytically about the system studied in this paper. Therefore, the parameter space involved is investigated largely by numerical experimentation.

One can imagine many ways of organizing such a bang-bang control system. The basic idea of the one chosen for this study is illustrated in Fig. 1. One can imagine the resistive wall (or part of it) covered by a number of independent systems. Each system consists of a circuit involving two closely coupled feedback coils located a little outside the resistive wall. A sensor coil is placed near them at the resistive wall measuring the magnetic field (associated with the mode in question) penetrating the wall. The switches, SW1 and SW2 are set so that the feedback coil currents tend to counteract the field detected by the sensor coil. The coil currents are driven by a dc power supply providing the constant voltage V. The switches, SW1 and SW2, are imagined arranged so that always one and only one of them is closed. The freewheeling diodes allow opening of the switches. The resistors serve the purpose of limiting the coil currents. The two feedback coils are assumed closely coupled, and the switches as well as the diodes are assumed ideal. It then follows, from the circuit equation, that the sum of the coil currents is constant,

$$
I_{1}+I_{2}=V / R \text {, }
$$

independent of how the switches are set. The field, produced by the feedback coils, is proportional to $I_{1}-I_{2}$. It is assumed that the field detected by the sensor coil, as well as its derivatives, is measured at times $T_{N}=N \times T_{S W}, N=0,1, \cdots$. Two prescriptions for setting the switches have been considered. For the time between $T_{N}$ and $T_{N+1}$ the switch setting may be determined by the sign of the sensor coil signal at $T_{N}$. The other possibility considered is to extrapolate the sensor signal from $T_{N}$ to $T_{N+1}$ using first and second derivatives at $T_{N}$. One may then let the switch setting for the period $T_{N}$ to $T_{N+1}$ be determined by the sign of the extrapolated field. 


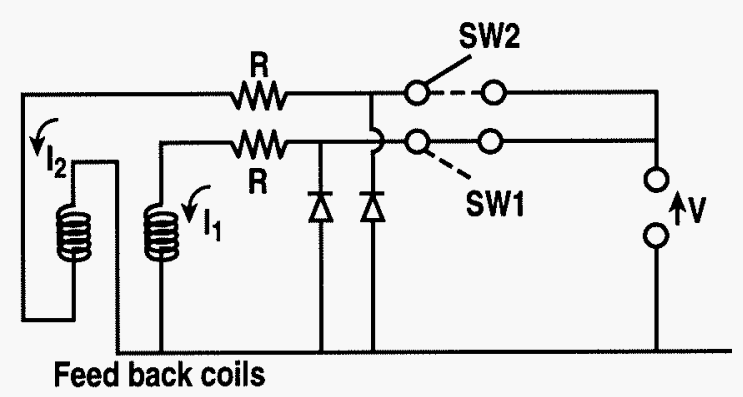

Fig. 1. Diagram of the current drive part of the control system. The solid or dashed lines of the switches, indicate the two possible switch positions.

For the analysis, presented in the following section, further simplifying assumptions are made, similar to those of Ref. 9. These assumptions may seem drastic, but the approach of Ref. 9 has proven useful as a guide for performing experiments as well as for interpretation of experimental results. The following Section III provides results found from numerical experimentation. In Section IV is considered how one might approach the problem of designing a bang-bang system for controlling inherently unstable resistive wall modes. In Section V, a general discussion of the subject of control systems for resistive wall modes is given. 



\section{ANALYSIS}

The key assumption is that the magnetic field of the mode considered, $\bar{b}$, satisfies the following geometric conditions

$$
\partial / \partial z=0, \partial / \partial y=i k, \bar{b}=\bar{\nabla} \phi \times \hat{z}
$$

For the tokamak case with the feedback system mainly located around the outboard midplane, $z$ is in the poloidal direction, $y$ in the toroidal direction and $x$ in the radial direction. The resistive wall is assumed located at $x=0$.

The currents, $J_{i}$, associated with the field of the mode in question are all assumed to be sheet currents in the $z$-direction and located in the $y$-z-plane. They are:

1. The plasma current, $J_{p}=A \phi(0)$ located at $x=-a$ ( $A$ is a constant)

2. The error current, $J_{e}$ located also at $x=-a$

3. The wall current, $J_{w}$ located at $x=0$

4. The feedback coil current, $J_{1}-J_{2}$, located at $x=b$.

The relationship between these currents and the flux function, $\phi$, is conveniently expressed using a Green's function

$$
\phi(x)=\frac{\mu_{o}}{2 k} \sum_{i} J_{i} e^{-k\left|x-x_{i}\right|},
$$

Here, $x_{i}$ is the location of the sheet current $J_{i}$, and the wave number $k$ is taken to be positive.

The quantity $A$ associated with the plasma current is real when the plasma is assumed ideal. As shown below, $A$ and $a$ can be expressed by the growth rate of the mode considered if no feedback system existed. The error field current, $J_{e}$ is included in the model to mimic the existence of a source for an error field, driven independently of the inherently unstable mode. For the wall current, $J_{w}$, it is assumed that the wall is so thin that its current only depends on the ratio of its thickness, $\delta$, and the resistivity of its material, $\eta$. 
The currents and the flux function, $\phi$, all have the $y$-dependence $e^{i k y}$. For the sake of brevity this $y$-dependence is omitted in the equations below. Also, the flux function is a function of time, $t$, and $x$. Since only two locations in $x$ are used, namely $x=0$ and $x=b$ the time dependence of $\phi$ as well as of the currents are not specifically indicated in the notation used.

The relationships between $\phi(0)$ and $\phi(b)$ and the currents are using Eq. (3)

$$
\begin{aligned}
& \phi(0)=\frac{\mu_{o}}{2 k}\left[A \phi(0) e^{-k a}+J_{e} e^{-k a}+J_{W}+\left(J_{1}-J_{2}\right) e^{-k b}\right], \\
& \phi(b)=\frac{\mu_{o}}{2 k}\left[A \phi(0) e^{-k a} e^{-k b}+J_{e} e^{-k a} e^{-k b}+J_{w} e^{-k b}+J_{1}-J_{2}\right] .
\end{aligned}
$$

The time dependence of the error current, $J_{e}$ is chosen, somewhat arbitrarily as discussed later. Its magnitude is related to the magnitude of the error field at the wall and normal to the wall, $b_{e}$, in the absence of other currents through

$$
b_{e}=\frac{1}{2} J_{e^{e^{-k a}}} \text {. }
$$

The wall current is given by

$$
J_{w}=-\frac{\delta}{\eta} \frac{d \phi(0)}{d t}
$$

Following Eq. (1), one has

$$
J_{1}+J_{2}=\frac{V_{e}}{R}
$$

Here $V_{e}$, the driving voltage (really an electric field with the unit volts per meter) and the resistance, $R$ are constants. Finally, the circuit equation (Fig. 1) for the current $J_{1},\left(I_{1}\right.$, in Fig. 1) is

$$
R J_{1}+\frac{d}{d t} \phi(b)=\left\{\begin{array}{l}
V_{\mathrm{e}} \text { if } \mathrm{SW} 1 \text { is closed } \\
0 \text { if SW } 1 \text { is open }
\end{array}\right.
$$

It is straightforward (albeit a bit tedious) from Eqs. (4) through (9) to obtain a second order equation in $\phi(0)$. For brevity, the following quantities are introduced:

$$
\tau_{w}=\frac{\mu_{o} \delta}{2 k \eta}
$$




$$
\alpha=\frac{\mu_{o}}{2 k} A e^{-k a}-1
$$

and

$$
Q=\frac{R \delta}{2 \eta}=\frac{R k \tau_{w}}{\mu_{o}} .
$$

Then one gets

$$
\begin{aligned}
& \frac{d^{2} \phi(0)}{d t^{2}} \tau_{w}\left(1-e^{-2 k b}\right)+\frac{d \phi(0)}{d t}\left[Q-\alpha+(1+\alpha) e^{-2 k b}\right] \\
& -\phi(0) \alpha Q / \tau_{w}-b_{e} \frac{Q}{\tau_{w} k}-\frac{1}{k} \frac{d b_{e}}{d t}\left(1-e^{-2 k b}\right) \\
& -\frac{V_{e}}{2} e^{-k b} S=0 .
\end{aligned}
$$

Here, $S=1$ when $S W 1$ (Fig. 1) is closed, $S=-1$ when $S W 1$ is open. It is further convenient to introduce the dimensionless flux function

$$
\Phi=\frac{\phi(0)}{V_{e} \tau_{w}},
$$

the dimensionless time

$$
\tau=\frac{t}{\tau_{w}}
$$

and the dimensionless error field

$$
F=\frac{b_{e} Q}{V_{e} k \tau_{w}}-\frac{1}{k V_{e}} \frac{d b_{e}}{d t}\left(1-e^{-2 k b}\right)
$$

Then Eq. (13) becomes

$$
\begin{aligned}
& \frac{d^{2} \Phi}{d \tau^{2}}\left[1-e^{-2 k b}\right]+\frac{d \Phi}{d \tau}\left[Q-\alpha+(1+\alpha) e^{-2 k b}\right]-\Phi \alpha Q \\
& -F-S \frac{e^{-k b}}{2}=0 .
\end{aligned}
$$

The times of potential resetting of the switch position are,

$$
\tau_{N}=N \tau_{S W} \quad, N=1,2, \cdots .
$$

The two prescriptions (discussed in Section 1) for the switch position considered, i.e., the quantity $S$ of Eq. (17) for $\tau_{N}<\tau<\tau_{N+1}$ are 


$$
S=\left\{\begin{array}{l}
\frac{\Phi\left(\tau_{N}\right)}{\left|\Phi\left(\tau_{N}\right)\right|} \\
\frac{\Phi\left(\tau_{N}\right)+\tau_{S W} \frac{d \Phi}{d \tau}\left(\tau_{N}\right)+\frac{1}{2} \tau_{S W}{ }^{2} \frac{d^{2} \Phi}{d \tau^{2}}\left(\tau_{N}\right)}{\mid \Phi\left(\tau_{N}\right)+\tau_{S W} \frac{d \Phi}{d \tau}\left(\tau_{N}\right)+\frac{1}{2} \tau_{S W}{ }^{2} \frac{d^{2} \Phi}{d \tau^{2}}\left(\tau_{N}\right)}
\end{array} .\right.
$$

The upper prescription of (19) is called "sign of $\Phi$ " criterion. The lower prescription is called “extrapolated sign of $\Phi "$ criterion. Thus, given initial values of $\Phi$ and $d \Phi / d \tau$, specifying $F(\tau)$ as well as choosing switch criterion, it is possible from Eqs. (17) and (19) to find $\Phi(\tau)$.

Consider first the case of no feedback system, i.e., $k b \rightarrow \infty$, and no error current, i.e., $F=0$. For this case one sees from Eq. (17) that the solution for $\Phi$ is of the form

$$
\Phi(\tau)=\Phi_{o} e^{\gamma \tau}
$$

with

$$
\gamma=\left\{\begin{array}{l}
\alpha \\
-Q
\end{array} .\right.
$$

Since the damped solution $(\gamma=-Q)$ is of no importance, one sees that $\alpha$ is the inherent growth rate of the resistive wall mode (normalized with the resistive wall time, $\tau_{w}$ ). Therefore, only $\alpha>0$ is of interest here.

One sees further from Eq. (17) that if (the terms with) $\Phi$ ever becomes large so that the term with $S$ can be ignored, control is lost; the amplitude of the mode will grow exponentially.

It seems a difficult problem to determine for given initial (small) values of $\Phi$ and $d \Phi / d t$ as well as given values of $Q, \alpha, k b, F(\tau)$ and the switch criterion Eq. (19) if control will ever be lost. The problem may be considered a mapping problem of $\Phi\left(\tau_{\mathrm{N}}\right), d \Phi / d \tau\left(\tau_{\mathrm{N}}\right), N=0,1,2, \ldots$ As part of the numerical experimentation, discussed in the following Section III a Poincaré map in the $\Phi\left(\tau_{\mathrm{N}}\right)-d \Phi\left(\tau_{\mathrm{N}}\right) / d \tau$ - plane was made for a numerical solution to Eqs. (17) and (19) for a period when control was maintained. The map had a complicated structure which seemed not to reveal any clues about when or if control would be lost eventually. 


\section{NUMERICAL RESULTS}

A numerical code was made for integrating Eqs. (17) and (19). In between switching times a simple algorithm for stepping forward in time was used:

$$
\begin{aligned}
& \Phi(\tau+\Delta \tau)=\Phi(\tau)+\Delta \tau \frac{d \Phi(\tau)}{d \tau}+\frac{1}{2} \Delta \tau^{2} \frac{d^{2} \Phi(\tau)}{d \tau^{2}} \\
& \frac{d \Phi}{d \tau}(\tau+\Delta \tau)=\frac{d \Phi(\tau)}{d \tau}+\Delta \tau \frac{d^{2} \Phi(\tau)}{d \tau^{2}} .
\end{aligned}
$$

$d^{2} \Phi / d \tau^{2}(\tau+\Delta \tau)$ was obtained from Eq. (17) using Eqs. (22) and (23). It was found sufficient to use $\Delta \tau=10^{-4}$.

The input parameters necessary to find $\Phi(\tau)$ are (besides initial values of $\Phi$ and $d \Phi / d \tau)$ :

1. The normalized distance from the resistive wall to the feedback coils, $k b$;

2. The normalized switch time, $\tau_{s w}$;

3. The inherent (normalized) growth rate of the resistive wall mode, $\alpha$;

4. The normalized resistance, $Q$;

5. The choice of time dependence and magnitude of the error field;

6. Choosing among the two switch criteria given by Eq. (19).

The most important result from a computer simulation of $\Phi(\tau)$ is for how long time control is maintained. As mentioned previously, when control is lost, it is never regained. It may be that in part of the input parameter space, control is maintained forever, while in other parts control is maintained only for a finite time. The time of maintaining control, $\tau_{\text {Loss }}$, seems not be a smooth function of the input parameters. An example was noted for which the same input parameters were used but by using single or double precision of the code, significantly different times of control were found. The somewhat arbitrary definition of maintenance of control used is

$$
\tau_{\text {LosS }}>10^{4}
$$

A measure of the quality of a given control system is for how large a value of the inherent growth rate, $\alpha$, control is maintained. Therefore, the object of the calculations is to find the 
maximum value of $\alpha, \alpha_{c r}$, which allows control. Since the parameter space is large, only a small part of it is investigated. The part investigated is believed relevant for a device such as DIII-D as discussed further in Section IV.

The parameter region considered is located around $k b=0.1, \tau_{s w}=0.02, Q=1.0$, $F=F_{o} \sin (0.1 \times \tau)$ with $F_{o}=0.1$. Only this slowly varying error field was considered.

Using the upper switch criterion of Eq. (19) the time of loss of control, $\tau_{\text {Loss }}$, defined as the time at which $|\Phi|>10^{-2}$, the results shown in Table I are obtained. According to the definition discussed above, for this case, $\alpha_{c r}=2.4$. Changing the switch criterion to the lower one of Eq. (19) ("sign of extrapolated $\Phi$ criteria") the results of Table II were obtained. From Table II one finds $\alpha_{c r}=14.7$. This shows that the "extrapolation criterion" provides a significantly better system than the "sign criterion". In a different region of parameter space with values of $Q \sim 10^{3}$, the opposite result was found. No explanation for this has been found.

TABLE I.

$\tau_{L O S S}$ AS A FUNCTION OF $\alpha$ FOR $k b=0.1, \tau_{S W}=0.02, Q=1.0, F_{o}=0.1$ AND "SIGN OF $\Phi "$ SWITCH CRITERION.

\begin{tabular}{ccccccccc}
\hline$\alpha$ & 1.0 & 2.0 & 2.3 & 2.4 & 2.5 & 3.0 & 5.0 & 12.0 \\
$\tau_{\text {LOSS }}$ & $>10^{4}$ & $>10^{4}$ & $>10^{4}$ & $>10^{4}$ & $5.8 \times 10^{2}$ & 5.2 & 3.7 & 0.5 \\
\hline \hline
\end{tabular}

TABLE II.

$\tau_{L O S S}$ AS A FUNCTION OF $\alpha$ FOR $k b=0.1, \tau_{S W}=0.02, Q=1.0, F_{o}=0.1$ AND “EXTRAPOLATED SIGN OF $\Phi "$ SWITCH CRITERION.

\begin{tabular}{ccccccc}
\hline$\alpha$ & 13.0 & 14.0 & 14.5 & 14.7 & 14.8 & 15.0 \\
$\tau_{\text {LosS }}$ & $>10^{4}$ & $>10^{4}$ & $>10^{4}$ & $>10^{4}$ & $2.5 \times 10^{3}$ & $1.0 \times 10^{3}$ \\
\hline \hline
\end{tabular}

The sensitivity of $\alpha_{c r}$ to the switch time, $\tau_{s w}$, was investigated varying $\tau_{s w}$ while the other parameters were kept at the central value, $k b=0.1, Q=1.0, F_{o}=0.1$ and the extrapolated switch criterion was used. The results are shown in Fig. 2. As expected, $\alpha_{c r}$ is a decreasing function of 
the switch time. One might think that $\alpha_{c r}$ should be approximately inversely proportional to $\tau_{s w}$, but that is not true for the cases of Fig. 2. In other parts of the parameter space it was found true.

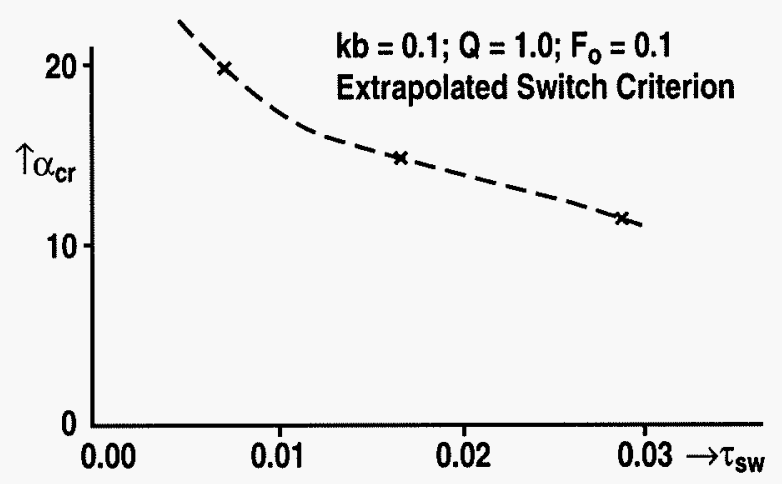

Fig. 2. The critical value of $\alpha, \alpha_{c r}$ vs. the switch time, $\tau_{s w}$.

In Fig. 3 is illustrated the sensitivity to the error field parameter, $F_{o}$. As expected, $\alpha_{c r}$ is a decreasing function of $F_{o}$. The time dependence of the error field (given above) was not varied.

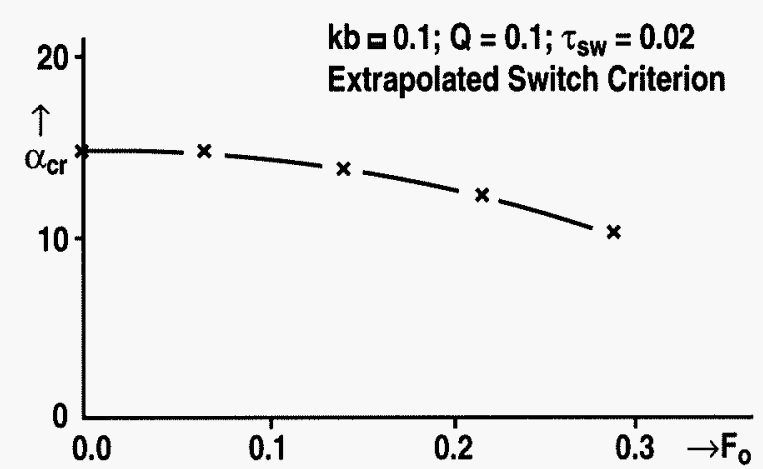

Fig. 3. The critical value of $\alpha, \alpha_{c r}$ vs. the error field amplitude, $F_{o}$.

Finally, the sensitivity to the normalized resistance, $Q$, was investigated numerically. The growth rate associated with the term containing $\Phi$ of Eq. (17), depends primarily on $\alpha$ and is not very sensitive to $Q$. From simple arguments one expects, however, to find an upper limit for $Q$ at which $\alpha_{c r}$ falls off since for large $Q$-values the S-term of Eq. (17) becomes small. For very small values of $Q$ one may guess from Fig. 1 that $J_{1} \sim J_{2}$ so that the effective feedback current, $J_{1}-J_{2}$ becomes small resulting in a small value of $\alpha_{c r}$. Numerical results on this issue are 
shown in Fig. 4. From other cases calculated, it was found that a sharp fall off of $\alpha_{c r}$ occurred above $Q=3000$.

The sensitivity of $\alpha_{c r}$ to $k b$ was not investigated. Similarly, only the above given time dependence of the error field was used.

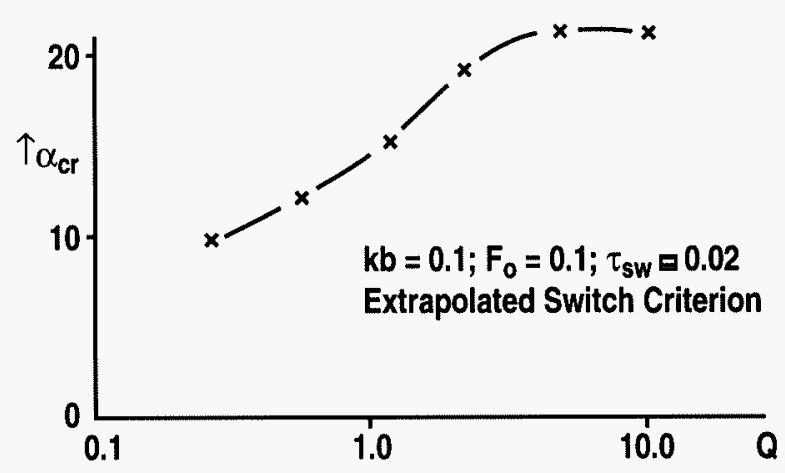

Fig. 4. The dependence of $\alpha_{c r}$ on the normalized resistance, $Q$. 


\section{DESIGN ISSUES}

The design of a feedback system of the type discussed involves, as usual, making complicated decisions involving desired performance on one hand (how large is $\alpha_{c r}$ ) and complications and cost on the other. The discussion of this section may be relevant for the DIII-D device (see Ref. 9). It is assumed that $k \sim 1 m^{-1}$ and $\tau_{w}=3 \times 10^{-3} s$, and that one may obtain $k b=0.1$. Furthermore, it may be considered acceptable to obtain $\alpha_{c r} \sim 10-20$ and reasonable to require immunity to error fields up to $b_{e}=3 \times 10^{-4} \mathrm{Vs} / \mathrm{m}^{2}$ [Eqs. (6) and (16)].

From the slow time dependence of the error field chosen one finds from Eq. (16)

$$
V_{e}=\frac{b_{e} Q}{F_{o} k \tau_{w}} \text {. }
$$

For the "central parameter point" chosen, $Q=1, F_{o}=0.1$ and $b_{e}=3 \times 10^{-4} \mathrm{Vs} / \mathrm{m}^{2}, \tau_{w}=3 \times 10^{-3} s$ as well as $k=1 \mathrm{~m}^{-1}$ one gets $\mathrm{V}=1 \mathrm{Volt} / \mathrm{m}$. For the currents of the system one gets using Eqs. (8), (12), and (25)

$$
J_{1}+J_{2}=\frac{b_{e}}{F_{o} \mu_{o}} \text {. }
$$

For the above used parameter values, one gets $J_{1}+J_{2}=2.4 \times 10^{3} \mathrm{~A} / \mathrm{m}$. The power consumed by the control system is approximately

$$
P \cong \frac{1}{2}\left(J_{1}+J_{2}\right) V_{e}=\frac{1}{2} \frac{b_{e}^{2} Q}{F_{o}^{2} \mu_{o} k \tau_{w}},
$$

which for the parameter values used above amounts to $1.2 \times 10^{3} \mathrm{~W} / \mathrm{m}^{2}$. This seems to be a reasonable consumption. Equation (27) illustrates the power penalty for requiring immunity towards error fields. From Fig. 4, one sees that performance can be improved by increasing $Q$, Eq. (27) illustrates the cost in increased power needs. Similarly, lower power requirements are achieved by increasing $F_{o}$, but at a cost of decreased performance as indicated by Fig. 3 .

It appears that "reasonably priced" integrated gate bipolar transistor (IGBT) switches may be used and that switching times of $60 \mu \mathrm{s}$, corresponding to $\tau_{s w}=0.02$, used for the "center" of 
parameter space are practical. The cost of the switches increases with decreasing switch times. Figure 2 may be of help deciding how to balance performance $\left(\alpha_{c r}\right)$ against switch costs.

For more detailed design work, it may be important to incorporate in the modeling that real diodes and switches have forward voltage drops.

The magnetic field penetrating the wall will, when the control system works, fluctuate around zero. Therefore, it is a relevant design question whether the amplitude of these fluctuations is tolerable or not. The root mean square of $\left.\Phi,<\Phi^{2}\right\rangle^{1 / 2}$ was calculated in the code. For the "central" point in the parameter space it was found that $\left\langle\Phi^{2}\right\rangle^{1 / 2} \approx 10^{-3}$. The root mean square of the fluctuating magnetic field penetrating the wall is then [use Eqs. (2) and (10)]

$$
<b_{1}^{2}>^{1 / 2}=<\Phi^{2}>^{1 / 2} V k \tau_{w} \text {. }
$$

For the parameter values considered above one finds $<b_{1}^{2}>^{1 / 2} \approx 3 \times 10^{-6} V s / m^{2}$. This is believed so small that it will not affect plasma performance. 


\section{DISCUSSION}

It is commonly believed that future fusion reactors will need a control system to prevent growth of resistive wall modes. Thus the cost of such systems may be of importance. To a lesser degree it may be important for experimental devices. Therefore, one should consider all available options. It seems that there are three types of control systems. One possibility involves the use of real linear amplifiers, such as audio amplifiers; this option was found attractive under circumstances where the power needed is relatively small ${ }^{12}$. In larger devices, such as DIII-D, pseudo-linear amplifiers are commonly used ${ }^{2}$ because they are cheaper than real linear (wideband width) amplifiers. The output of such amplifiers are by a bang-bang control system made to be proportional to an input signal; thus, they appear to be linear amplifiers. The problem is that the bandwidth of such amplifiers tends to be relatively small whereby the control ability of the system becomes limited. The third option is a bang-bang system. Using similar switch devices as in a pseudo-linear amplifier used for conventional control and a bang-bang system, it seems reasonable to expect that the bang-bang system may be more effective because the complexity of making a bang-bang system appear linear is avoided.

Control systems using real linear or pseudo-linear amplifiers have the advantage over bangbang systems that they can be described by linear models. In contrast, bang-bang systems are inherently nonlinear and mathematically complicated. Thus, the usual concept of stability cannot be used and as discussed above some uncertainty exists on where the boundary between acceptable and unacceptable control lies. It is believed, however, based on the numerical experiences gained from the present work, that this difficulty is mostly a mathematical and not a real one.

It is believed that there are many unexplored ways of making bang-bang control systems. The one considered in this paper should just be considered one example, and as pointed out in Section IV more work would be required before an engineering design should be made for it. To 
the author's knowledge, bang-bang control systems have not been seriously considered for the control of resistive wall modes, even though laboratory control of such modes have been employed for many years.

For conventional linear control systems, the power required before the amplifiers become saturated is determined by noise considerations. This is also the case for bang-bang systems. In the treatment in this paper the "error field" plays the role of noise, and as discussed in Section IV it plays an important role for the design. 


\section{REFERENCES}

[1] C.M. Bishop, Plasma Phys. Contr. Fusion 31, 1179 (1989).

[2] A.M. Garofalo, T.H. Jensen, L.D. Johnson, et al., Phys Plasmas 91997 (2002).

[3] R. Fitzpatrick and T.H. Jensen, Phys. Plasmas 3, 2641 (1996).

[4] T.H. Jensen and R. Fitzpatrick, Phys. Plasmas 4, 2997 (1997).

[5] M. Okabayashi, N. Pomphrey, and R.E. Hatcher, Nucl. Fusion 38, 1607 (1998).

[6] A.H. Boozer, Phys. Plasmas 5, 3350 (1998).

[7] T.H. Jensen and A.M. Garofalo, Phys. Plasmas 6, 2757 (1999).

[8] Q. Liu and A. Bondeson, Phys. Plasmas 7, 3681 (2000).

[9] A.M. Garofalo, T.H. Jensen, and E.J. Strait, Physics of Plasmas 94573 (2002).

[10] W. Levine (Ed), The Control Handbook, CRC Press, Boca Raton, 773 (1996).

[11] Y-C Tian, M.O. Tadé, and D. Levy, Physics Letters A 29687 (2002).

[12] C. Cates, M. Shilov, M.E. Mauel, et al., Phys. Plasmas 73133 (2000). 



\section{ACKNOWLEDGMENTS}

This is a report of work supported by the U.S. Department of Energy under Grant No. DE-FG03-97ER54309. The author appreciates enlightening and enjoyable discussions on the subjects of this paper with A. M. Garofalo in general, T. E. Evans specifically on the issues concerning the map of $\Phi$ and $d \Phi / d \tau$ for successive switching, and A. Nerem on the issue of switches available. Gary Jackson made several valuable suggestions which resulted in improvements of the paper. 